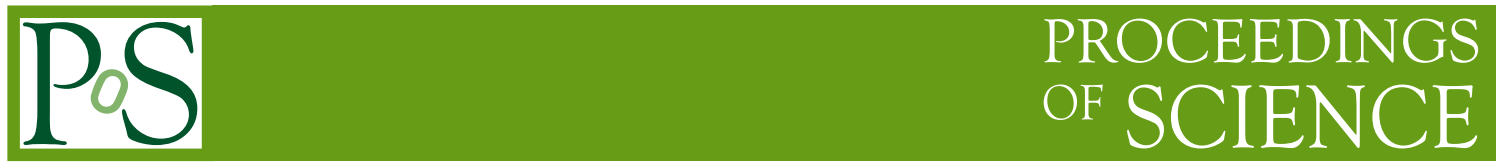

\title{
$\mathrm{X}$ Per pulse period evolution
}

\section{Pere Blay*i}

Image Processing Laboratory, University of Valencia, Valencia, Spain

E-mail: pere.blayduv.es

\section{Víctor Reglero}

Image Processing Laboratory, University of Valencia, Valencia, Spain

E-mail: Victor.reqleroduv.es

The Be X-Ray Binary system X Per has been observed by INTEGRAL/IBIS/ISGRI repeatedly during its 10 years of operations. The source shows an interesting spectral and pulse-period variability in the high energy range which will be discussed in detail. A continuous spin-up of the source with sporadic sudden changes in pulse period are reported. The possible correlation between timing parameters and spectral parameters is also reviewed. X Per is a BeX system with an orbital period of $250 \mathrm{~d}$ and a pulse-period of $836 \mathrm{~s}$. The source has been reported to show a CRSF in absorption around $30 \mathrm{keV}$ (see [罒) on a high energy spectrum well represented by a powerlaw or a thermal bremsstrahlung model (see []], and references therein) The OSA9 software was used to extract light curves and spectra from all public observations of the source up to revolution 970 .

"An INTEGRAL view of the high-energy sky (the first 10 years)" 9th INTEGRAL Workshop and celebration of the 10th anniversary of the launch,

October 15-19, 2012

Bibliotheque Nationale de France, Paris, France

\footnotetext{
* Speaker.

†The author acknowledges support from the spanish Plan Nacional de Espacio through project AYA 2011-29936C05-01.
} 


\section{Introduction}

BeX systems are stellar binary systems consisting of a Be star and a neutron star orbiting each other. A Be star is a main sequence B star which developes a circunstellar equatorial decretion disk which is responsible of the visible emission lines present in the optical spectrum of the star and the excess observed in its infrared magnitudes, with respect normal B stars [6]. The neutron star companion accretes matter from the slow and dense wind which forms the equatorial structure around the B star, leading the accretion phenomena responsible of the high energy emission detected in these sources.

$\mathrm{X}$ Per is a BeX system, with an orbital period of $\sim 250 \mathrm{~d}$ and a pulsed X-ray emission with a periodicity of $\sim 836 \mathrm{~s}$. As other accretion pulsars, the spectral and timing properties of $\mathrm{X}$ Per are related to the accretion regime taking place in the system. In this paper we review mainly the short term timing behaviour of this source (pulse period evolution) as deduced from INTEGRAL/ISGRI observations. Spectral properties are also reviewed briefly and compared to the timing observables, with the aim of searching for correlations. The ISDC OSA9 software ${ }^{1}$ was used to extract light curves and spectra from all public observations of the source up to revolution 970.

\section{INTEGRAL/ISGRI detections}

X Per has been repeatedly observed by INTEGRAL, specially from 2003 on, previously INTEGRAL/ISGRI detections of this source were only marginal [四].

Figure $\square$ shows the light curve of the source as detected by INTEGRAL/ISGRI in 4 different energy bands, namely $20-40 \mathrm{keV}, 40-60 \mathrm{keV}, 60-80 \mathrm{keV}$, and 80-100 keV. For the latter two intervals only upper limits are shown, as there are no detections above the $3 \sigma$ level (or detection level above 7, as reported by OSA 9 software ${ }^{\text {I }}$ ).

\section{Pulse period analysis}

Figure shows the light curve of the $20-40 \mathrm{keV}$ energy band as detected by INTEGRAL/ISGRI (lower panel) together with the spin period measurements obtained from a refined bin (250 s) of the same light curve. X Per was in a spin-down phase prior to the INTEGRAL observations reported here (see [䧃]). INTEGRAL/ISGRI caught X Per in a spin-up phase (also reported by [四]). The spin up rate obtained from these measurements, $3.8 \times 10^{-4} y r^{-1}$, is compatible with that obtained by [四]. However we can not confirm the plateau reported by them in INTEGRAL data. Although the errors are large, we notice the possible presence of a glitch around MJD 54730. If it can be confirmed by detailed further analysis, it would be the first glitch detected in this system. Other BeX systems in which glitches have been detected are KS 1947+300 (see [B] ) an SAX J2103.5+4545 (see [四]).

The pulse period evolution of X Per is complex and demands a detailed analysis and follow up for a better understanding.

We can investigate the evolution of the pulse period derivative by the use of a view graph like the one in Figure 3 . The area around MJD 54600 corresponds to an epoch of lower flux and the data errors are larger, on the order of $10^{-3} \mathrm{yr}^{-1}$, while the typical error for the rest of epochs is

\footnotetext{
${ }^{1}$ http://www.isdc.unige.ch/integral/analysis
} 


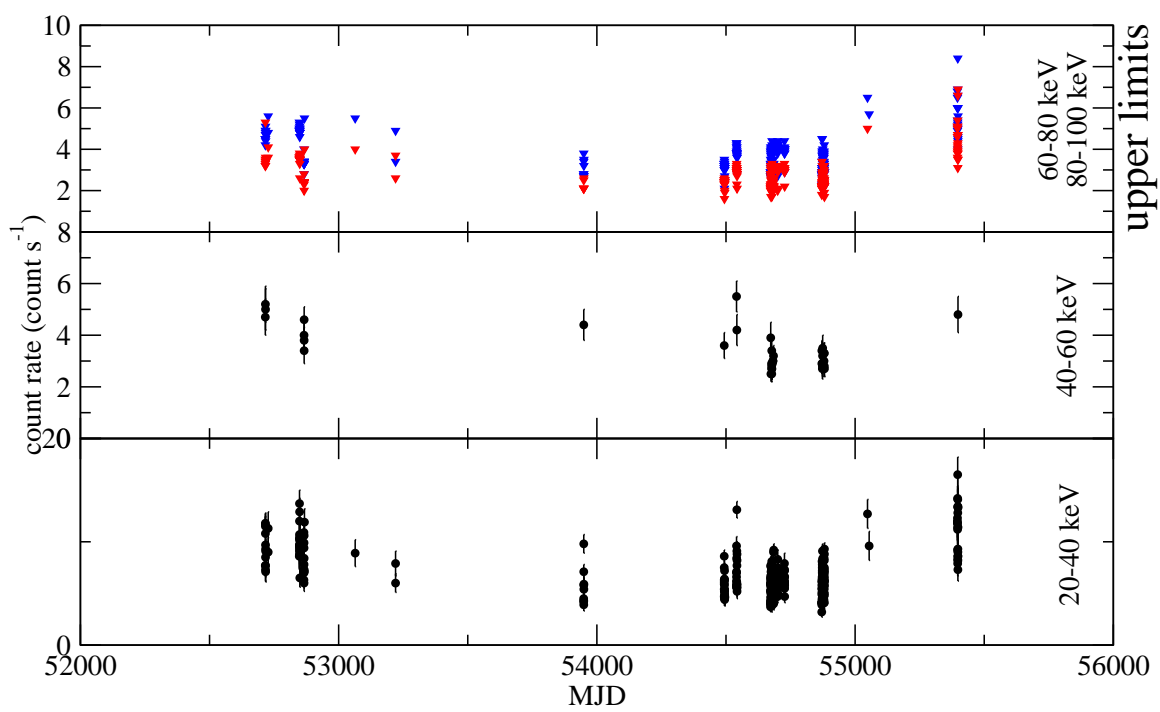

Figure 1: Long term X Per light curve.

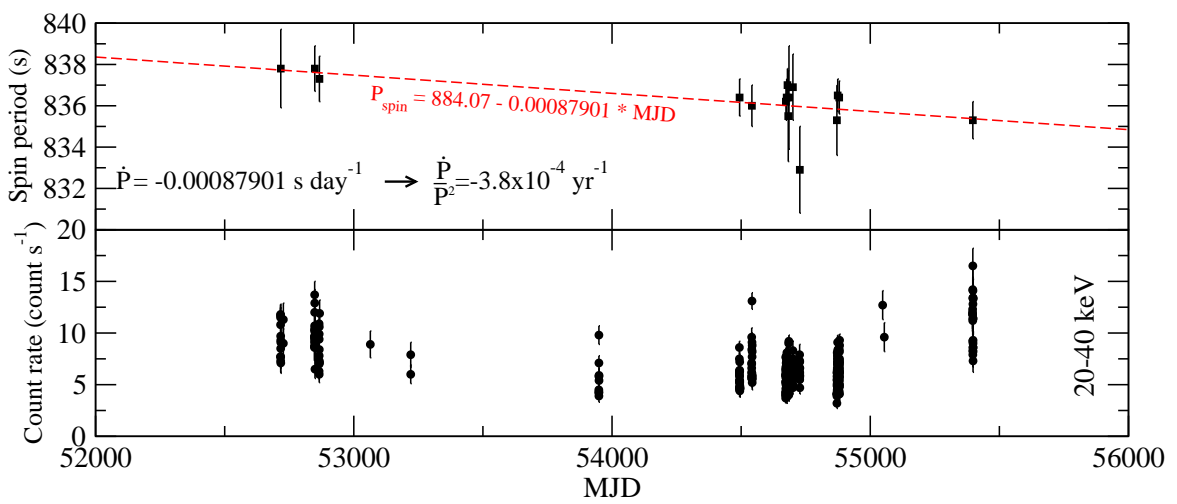

Figure 2: Evolution of the spin period of $X$ Per compared to the light curve in the $20-40 \mathrm{keV}$ energy band.

on the order of $10^{-6} \mathrm{yr}^{-1}$. Therefore, care must be taken when interpreting the high variability of $\dot{P}$ and $\dot{P} / P$ at this epoch, as the large error bars make this data compatible with the general trend indicated by the slashed line.

The pulse period derivative changes, according to this view graph, at a rate of $6.88 \times 10^{-6} y r^{-2}$, however this is only a rough estimation, as the correlation coefficient of the linear trend is not very good $\left(r^{2}=0.41\right.$, very likely due to the large scatter around MJD 54600). This result would imply a spin second derivative of $\ddot{P} / P \sim 7 \times 10^{-6} y r^{-2}$.

\section{Spectral Analysis}

In the left panel of Figure $\mathbb{A}$, the evolution of the spectral photon index with respect to the source flux is depicted. A powerlaw model was used to fit the spectral data. We can clearly see that the source becomes softer as the flux increases, although not linearly. A cyclotron resonant 


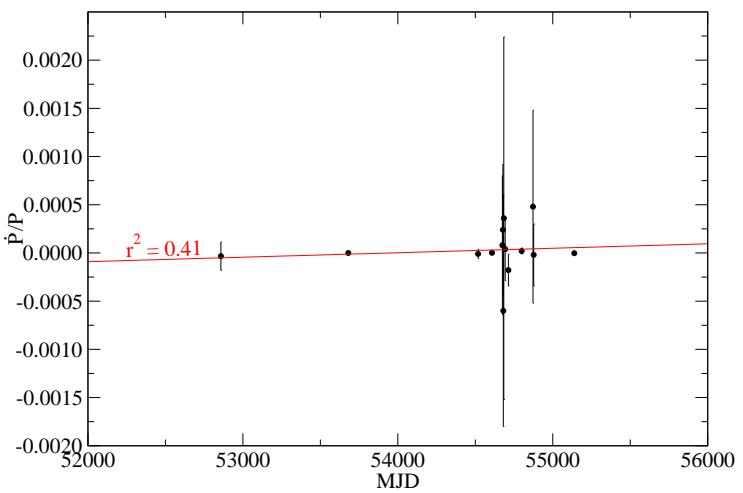

Figure 3: Evolution of $\dot{P}$.

scattering absorption feature (CRSF) (see [5] ) has been added to the spectral model by adding a Gaussian absorption. The determination of the central energy of the CRSF and its properties are not very well constrained by INTEGRAL/ISGRI spectra. The evolution of the line energy with the source flux is shown in the right panel of Figure 4 . No dependence of the line energy on the source flux or other spectral parameters can be inferred from this plot. An average spectrum per revolution has been used in order to improve the statistics with respect to individual spectra.
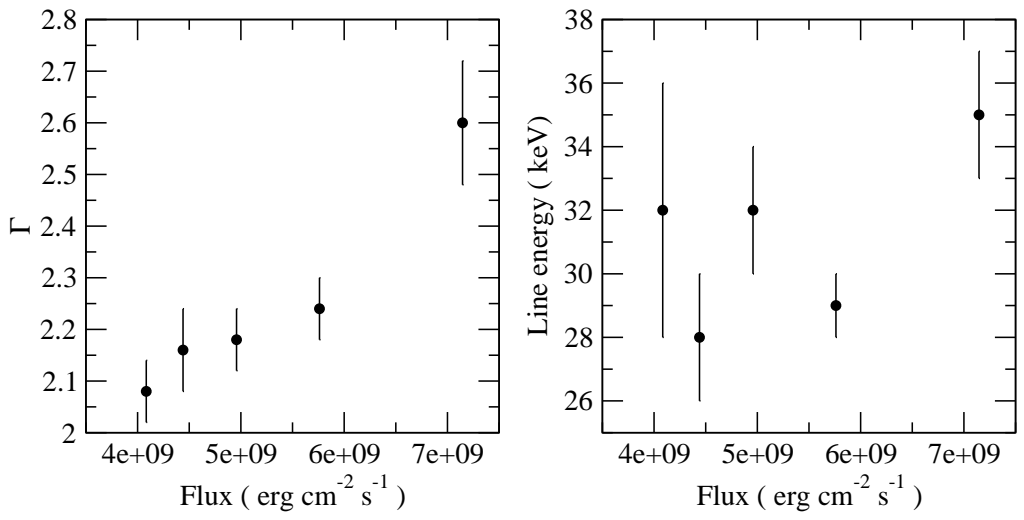

Figure 4: Spectral parameters versus $20-40 \mathrm{keV}$ flux.

\section{Discussion and conclusion}

$\mathrm{X}$ Per is a peculiar BeX system. Unlike other BeX systems, X Per, due to its large orbital size, is likely accreting from the slow wind of the Be star, rather than directly from its equatorial disk (see [D] ). Furthermore the X Per spin period is one of the longest for a BeX system. The long term timing behaviour of BeX systems are thought to be due to changes in the wind or disk structure of the Be star (see [焑]). INTEGRAL/ISGRI data shows no significant detection before 2003, but several detections afterwards. Therefore this may be an indication of large structural changes in the long term in the optical counterpart of X Per. 
The spin period of X Per is changing continuously, as reported here, in [团], and []. During the spin-up phase the spin period evolution of X Per is reported by [四] to show a plateau, and marginal evidence of a glitch is reported in this work. This irregular spin evolution reflects the properties of the non-homogeneous source of the accreted matter and its irregular flow in short time scales.

\section{References}

[1] Blay P., Camero A., Connell P., Reig P., Martínez-Nuñez S., Reglero V., 2008, PoS(INTEGRAL08)078

[2] Doroshenko V., Santangelo A., Kreykenbohm I., Doroshenko R., 2012, A\&A, 540, L1

[3] Galloway D. K., Morgan E. H., Levine A. M., 2004, ApJ, 613, 1164

[4] Lutovinov A, Tsygankov S., Chernyakova M., 2012, MNRAS, 423, 1978

[5] Nelson R.W., Wang J.C.L., Salpeter E.E., Wasserman I., 1995, ApJ, 438, L99

[6] Reig P., 2011, Ap\&SS, 332, 1

[7] Stark M.J., Heffner C M., Baykal A., Swank J.H., 2004, AAS, 205, 8303 